\title{
On spontaneous scalarization
}

\author{
Marcelo Salgado*, Daniel Sudarsky \\ Instituto de Ciencias Nucleares \\ Universidad Nacional Autónoma de México \\ Apdo. Postal 70-543 México 04510 D.F, México \\ and \\ Ulises Nucamendi \\ Departamento de Física \\ Centro de Investigación y de Estudios Avanzados del I.P.N. \\ A. P. 14-741, México, D. F. 07000, México.
}

\section{Abstract}

We study in the physical frame the phenomenon of spontaneous scalarization that occurs in scalar-tensor theories of gravity for compact objects. We discuss the fact that the phenomenon occurs exactly in the regime where the Newtonian analysis indicates it

should not. Finally we discuss the way the phenomenon depends on the equation of state used to describe the nuclear matter.

PACS number(s): 04.50.+h, 97.60.Jd, 04.25.Dm

*e-mail: marcelo@nuclecu.unam.mx 


\section{INTRODUCTION}

In recent years, alternative field theories of gravity (scalar-tensor theories) have been analyzed in many contexts (see [1] for a review), specially as a way to study possible deviations from general relativity and trough comparison with experimental results put bounds on the magnitude of such deviations.

The basic assumption of these theories is the existence of a single or multiple fundamental scalar fields which are perhaps the effective relics of a fundamental unified theory like superstrings, supergravity or Kaluza-Klein theory, and that might couple to gravity either minimally or non-minimally.

One of the most interesting effects occurring in a certain class of scalar-tensor theories of gravity is the phenomenon of spontaneous scalarization in neutron stars that was recently discovered by Damour and Esposito-Farèse [2,3], which consists in the appearance of a non-trivial configuration of a scalar field in the absence of sources and with vanishing asymptotic value. This phenomenon requires the presence of a scalar field nonminimally coupled to gravity. This nonminimal coupling can be thought as a field-dependent gravitational constant in the spirit of the Brans-Dicke theory [4 [7]. The occurrence of this highly nonlinear effect can be used to put very stringent limits on the deviations from general relativity, and in fact using the binary pulsar, the limits for certain range of parameters seem to be more severe than those that can be inferred from solar system experiments [3.

One puzzling aspect of the phenomenon is that occurs exactly in the regime where the Newtonian analysis indicates it should not, while on the other hand, no such phenomenon has been said to occur in the regime where the Newtonian analysis indicates it should. We give a very short description of these facts based on an energetic analysis and then carry out a numerical study to examine its validity and explain why does the full fledge theory behave in the opposite direction as compared to what is indicating by the Newtonian intuition. To do this we have to perform the analysis in the physical frame where a counterpart to the intuitive Newtonian ideas can be found in contrast with the original analysis [2.3], which was carried out in a conformal frame that, although yield simpler equations, corresponds to a description that is less susceptible of comparison with the Newtonian analysis.

Finally, and in view of the fact that the phenomenon when applied to the binary pulsar is being used to put severe limits on the theory's parameters, we investigate the degree to which the phenomenon depends on the equation of state assumed for the description of the nuclear matter of neutron stars.

The paper is organized as follows. In section II we briefly review the phenomenon of spontaneous scalarization. In section III we provide the naive Newtonian energetic analysis of spontaneous scalarization. In section IV we introduce the scalar-tensor theory we will analyze in detail, derive the equations of motion for a spherically symmetric, static space-time, and make some comments about the realistic equations of state used in this work. In section $\mathrm{V}$ we discuss the boundary conditions for the numerical integration, and define the global quantities to be computed and which are of astrophysical interest. 
In section VI we provide the numerical analysis and the explanation of the origin of spontaneous scalarization on relativistic energetic grounds. Finally, in section VII we discuss and summarize our results.

\section{SPONTANEOUS SCALARIZATION}

As we mentioned, the phenomenon of spontaneous scalarization was discovered by Damour and Esposito-Farèse in scalar-tensor theories of gravity [2:3], and further studied in the context of stability analysis [9:[10]. However, we have to comment that Zagaluer [8] had previously argued for large deviations from general relativity in neutron stars in a particular class of scalar-tensor theories. Such results have been criticized in that seem spurious due to an artificial definition of some parameters [2].

In these theories, gravity is described both by the standard type $(0,2)$ tensor field, and by one or more scalar fields. We concentrate for simplicity in the case of one scalar field, and the Lagrangian is then taken to be:

$$
\mathcal{L}=\left(\frac{1}{16 \pi G_{0}}\right) \sqrt{-g}\left[f(\phi) R-z(\phi)(\nabla \phi)^{2}\right]+\mathcal{L}_{\text {mat }}\left(\psi, g_{\mu \nu}\right) .
$$

Where $G_{0}$ is the Newton's gravitational constant, $\psi$ denotes the matter fields, and $g_{\mu \nu}$ stands for the physical metric (Jordan metric). Using the conformal transformation to a non-physical metric $g_{\mu \nu}^{*}=f(\phi) g_{\mu \nu}$ (Einstein metric) and redefining a new scalar field,

$$
\varphi=\int\left[\frac{3}{4} \frac{1}{f^{2}(\phi)}\left(\frac{\partial f(\phi)}{\partial \phi}\right)^{2}+\frac{1}{2} \frac{z(\phi)}{f(\phi)}\right]^{1 / 2} d \phi,
$$

this Lagrangian can be brought in the form

$$
\mathcal{L}=\left(\frac{1}{16 \pi G_{0}}\right) \sqrt{-g_{*}}\left[R_{*}-2\left(\nabla_{*} \varphi\right)^{2}\right]+\mathcal{L}_{\text {mat }}\left(\psi, f^{-1}(\varphi) g_{\mu \nu}^{*}\right) .
$$

Here the tensorial operations are performed by using the non-physical metric $g_{\mu \nu}^{*}$. The reason given in [2, [3] for writing the field equations in terms of the non-physical metric rather than the physical one is the mathematical simplicity related to the fact that the non-physical gravitational variables, $g_{\mu \nu}^{*}$ and $\varphi$, are minimally coupled. Many authors have also employed that frame to analyze neutron-star equilibrium configurations in other related contexts [9 [12].

The field equations derived of the above Lagrangian are

$$
\begin{gathered}
G_{\mu \nu}^{*}=2 \nabla_{\mu}^{*} \varphi \nabla_{\nu}^{*} \varphi-g_{\mu \nu}^{*}\left(\nabla_{*} \varphi\right)^{2}+8 \pi G_{0} f^{-1}(\varphi) T_{\mu \nu} \\
\square^{*} \varphi=2 \pi G_{0} f^{-2}(\partial \ln f / \partial \varphi) T, \\
\nabla_{\mu} T^{\mu \nu}=0
\end{gathered}
$$


where $T^{\mu \nu}$ is the physical stress-energy tensor. For a particular class of scalar-tensor theories and using this frame (Einstein frame), the effects on neutron stars were investigated. Specifically, it was analyzed the equilibrium configurations of a spherically symmetric, static, isolated neutron stars using polytropic equations of state to model the nuclear matter [2,9-12], and also in axisymmetric slow rotating polytropes [3].

In particular, it was found that for the case of scalar-tensor theories with $\beta\left(\varphi_{0}=0\right) \leq$ $-4\left[\right.$ where $\beta(\varphi)=(-1 / 2)\left(\partial^{2} \ln f / \partial \varphi^{2}\right)$ ], there existed a certain critical baryonic mass above which the star developed a non-zero scalar field $\varphi$, even if $\varphi_{0}=0$ (here $\varphi_{0}$ is the value at spatial infinity of the scalar field, i.e., the cosmological value) [2, 3, 9]. This occurs because such configurations are energetically more favorable than the one having $\varphi=0$ in all the space (both configurations having equal and constant baryonic masses). This striking effect was baptized as spontaneous scalarization in analogy with the spontaneous magnetization arising in ferromagnets below the Curie temperature. These equilibrium configurations lead to strong deviations from general relativity in the dynamics of binarypulsar systems [3]. The fact that the analysis was done in the non-physical frame does not allow us to understand the interplay of the true contributions to the energy due to the physical fields that are responsible of this phenomenon. We refer the reader to Ref. 13. for a discussion of the problems associated with this point.

\section{NEWTONIAN ENERGETIC ANALYSIS}

One would like to understand the occurrence of the phenomenon of spontaneous scalarization in neutron stars on simple energetic grounds, much in the same way that one understands the occurrence of spontaneous magnetization on ferromagnetic materials by comparing the free energy of the states with no magnetization to the free energy of the states with magnetization arriving to the conclusion that for a sufficiently low temperature the free energy is minimized by the latter rather than the former.

Let's try then to understand the phenomenon on these energetic terms using a Newtonian analysis of the contributions to the total energy of a given matter-scalar field configuration. First of all we recall that one expects the true equilibrium configurations in this case to correspond to the minima of the energy within the space of configurations with fixed values of the other conserved quantities [14], in this case the only relevant conserved quantity is the total baryon number of the neutron star (for simplicity we are taking the temperature to vanish). From this point of view one would think naively that since the net contribution of the scalar field to the energy is expected to be positive, the minimizing configuration should have $\phi \equiv 0$. A little thought then points to a caveat given the fact that in the class of scalar-tensor theory with $f(\phi)=\left(1+16 \pi \xi \phi^{2}\right)$, the effective gravitational constant depends on $\phi$, i.e., $G_{\text {eff }}=G_{0} /\left(1+16 \pi \xi \phi^{2}\right)$. The point is that we expect the total energy of the configuration to be given by the sum of the mass energy of the neutrons (neglecting any thermal energy and those contributions arising from short range interactions), the energy of the scalar field, and the gravitational binding energy. Thus roughly we expect 


$$
\mathcal{M}=\int \rho_{\mathrm{bar}}(\bar{x}) d^{3} \bar{x}+\int \rho_{\phi}(\bar{x}) d^{3} \bar{x}-G_{\mathrm{eff}}(\phi) \int \frac{\rho_{\mathrm{bar}}(\bar{x}) \rho_{\mathrm{bar}}(\bar{y})}{|\bar{x}-\bar{y}|} d^{3} \bar{x} d^{3} \bar{y}+\ldots
$$

where we took into account the dependence of the effective gravitational constant on $\phi$. Now we recall that we are expected to minimize $\mathcal{M}$ subject to the condition

$$
\mathcal{M}_{\text {bar }}=\int \rho_{\text {bar }}(\bar{x}) d^{3} \bar{x}=\quad \text { constant }
$$

Thus the first term in Eq. (7) is fixed, and the second is expected to be positive, but it is conceivably that if the third integral is sufficiently large (i.e., if the object is compact enough) then the positive energy contribution of the scalar field can be more than compensated by the change in the $G_{\text {eff }}$ that would result in the gravitational binding energy becoming substantially more negative, and thus making the configurations with a non-vanishing scalar field energetically preferred. This, of course, would require that $G_{\text {eff }}$ be an increasing function of $\phi$, which in our specific case corresponds to $\xi<0$. Thus we seem to have arrived to the conclusion that the phenomenon of spontaneous scalarization is possible only if $G_{\text {eff }}$ be an increasing function of $\phi$. This is precisely the opposite of what is found in the relativistic analysis carried out in Refs. [2, 3], and in the present paper.

We will examine next the phenomenon using the exact form of the theory in order to understand why did the naive Newtonian analysis fail so miserably.

\section{FORMULATION OF THE MODEL}

We will consider a model of a scalar field $\phi$ non-minimally coupled (NMC) to gravity. One of the simplest models of this kind is obtained by considering the Lagrangian

$$
\mathcal{L}=\left(\frac{1}{16 \pi G_{0}}+\xi \phi^{2}\right) \sqrt{-g} R-\sqrt{-g}\left[\frac{1}{2}(\nabla \phi)^{2}+V(\phi)\right]+\mathcal{L}_{\text {mat }}
$$

Here $\xi$ stands for the NMC constant, and $V(\phi)$ is a scalar potential. In this model the schematic matter Lagrangian $\mathcal{L}_{\text {mat }}$ is intended to represent the ordinary matter contribution to the total Lagrangian.

As we mentioned, equation (9) shows that the introduction of the coupling term is equivalent to consider an effective gravitational constant which explicitly depends on the scalar field. We note that for $\xi$ positive, (the convention that we keep in all the paper), the effective gravitational constant might only decrease with respect to the Newtonian value:

$$
G_{\mathrm{eff}}=\frac{G_{0}}{1+16 \pi G_{0} \xi \phi^{2}}
$$

The gravitational field equations following from the Lagrangian (9) can be written as

$$
R^{\mu \nu}-\frac{1}{2} g^{\mu \nu} R=8 \pi G_{0} T_{\text {eff }}^{\mu \nu}
$$


where

$$
\begin{aligned}
& T_{\mathrm{eff}}^{\mu \nu}=\frac{G_{\mathrm{eff}}}{G_{0}}\left(4 \xi T_{\xi}^{\mu \nu}+T_{\mathrm{sf}}^{\mu \nu}+T_{\mathrm{mat}}^{\mu \nu}\right) \\
& T_{\xi}^{\mu \nu}=\nabla^{\mu}\left(\phi \nabla^{\nu} \phi\right)-g^{\mu \nu} \nabla_{\lambda}\left(\phi \nabla^{\lambda} \phi\right) \\
& T_{\mathrm{sf}}^{\mu \nu}=\nabla^{\mu} \phi \nabla^{\nu} \phi-g^{\mu \nu}\left[\frac{1}{2}(\nabla \phi)^{2}+V(\phi)\right] .
\end{aligned}
$$

The energy-momentum tensor of matter $T_{\text {mat }}^{\mu \nu}$ will be represented by a perfect fluid describing the cold catalyzed matter of a neutron star [15]:

$$
T_{\mathrm{mat}}^{\mu \nu}=(p+\rho) U^{\mu} U^{\nu}+p g^{\mu \nu}
$$

which possesses the symmetries of the spacetime. The scalar field will also be assumed to posses these symmetries.

Finally, the equation of motion for the scalar field becomes

$$
\square \phi+2 \xi \phi R=\frac{\partial V(\phi)}{\partial \phi}
$$

We will focus on a metric describing spherical and static space-times. Four our purposes it will be convenient to adopt the so-called radial gauge coordinates which are also maximal slicing coordinates (hereafter we refer these coordinates as to RGMS coordinates):

$$
d s^{2}=-N^{2}(r) d t^{2}+A^{2}(r) d r^{2}+r^{2} d \theta^{2}+r^{2} \sin ^{2} \theta d \varphi^{2}
$$

Our goal is to analyze solutions of the gravitational, matter, and scalar field equations describing neutron-star models and the resulting space-time. Owing to the complexity of the resulting equations, we will perform a numerical analysis. To do so it will be helpful to adopt the following variables

$$
\begin{aligned}
\nu(r) & =\ln [N(r)] \\
\tilde{\nu}(r) & =\nu(r)-\nu(0), \\
A(r) & =\left(1-\frac{2 G_{0} m(r)}{r}\right)^{-1 / 2} .
\end{aligned}
$$

The relevant Einstein equations take then the following form

$$
\begin{aligned}
& \frac{\partial m}{\partial r}=4 \pi r^{2} E \\
& \frac{\partial \tilde{\nu}}{\partial r}=A^{2}\left\{\frac{G_{0} m}{r^{2}}+4 \pi r G_{0} T_{\text {eff } r}^{r}\right\} .
\end{aligned}
$$

where

$$
E=N^{2} T_{\mathrm{eff}}^{t t}
$$


is the effective total energy density.

On the other hand, the Klein-Gordon equation can be written directly in terms of sources as follows:

$$
\square \phi=-16 \pi \xi \phi G_{0}(E-S)+\frac{\partial V(\phi)}{\partial \phi}
$$

where

$$
S=T_{\text {eff } i}^{i}
$$

is the trace of the spatial part of $T_{\text {eff }}^{\mu \nu}$, which plays the role of an effective pressure.

In the RGMS coordinates this equation reads

$$
\begin{aligned}
\frac{\partial^{2} \phi}{\partial r^{2}}= & -\left[\frac{2}{r}+\frac{\partial \tilde{\nu}}{\partial r}-\left(1-\frac{2 G_{0} m}{r}\right)^{-1}\left(4 \pi G_{0} r E-\frac{G_{0} m}{r^{2}}\right)\right] \frac{\partial \phi}{\partial r} \\
& +\left(1-\frac{2 G_{0} m}{r}\right)^{-1}\left[\frac{\partial V(\phi)}{\partial \phi}-16 \pi \xi \phi G_{0}(E-S)\right] .
\end{aligned}
$$

The matter equations are obtained from

$$
\nabla_{\mu} T_{\mathrm{eff}}^{\mu \nu}=0
$$

and straightforward manipulations with help of the Einstein equations show that the matter and the scalar-field energy-momentum tensors are conserved separately, leading thus to

$$
\nabla_{\mu} T_{\mathrm{matt}}^{\mu \nu}=0
$$

This yields the equation of hydrostatic equilibrium which is similar to the one obtained in general relativity with no scalar fields.

For cold catalyzed matter (see below), it's customary to have the equations of state (EOS) parameterized by the baryon density $n(r)$, thus it is convenient to write the equation of hydrostatic equilibrium in terms of this:

$$
\frac{\partial n}{\partial r}=-\frac{(\rho+p) n}{\gamma p} \frac{\partial \tilde{\nu}}{\partial r}
$$

where $\gamma:=\frac{d \ln [p]}{d \ln [\rho]}$ is the adiabatic index.

It is clear from Eqs. (12 14), that the intermediary variables $E$ and $S$ [see Eqs.(23), (25)] involve second order derivatives of the scalar field. However, we can eliminate such a terms from the gravitational field equations with the help of the Klein-Gordon equation, and obtain "sources" containing at most first order derivatives of the scalar field. We also introduce the following dimensionless quantities (where we restore the factors of $c$ ) 


$$
\begin{aligned}
\tilde{r} & :=r \cdot \frac{c^{2}}{G_{0} M_{\odot}}, \\
\tilde{m} & :=\frac{m}{M_{\odot}}, \\
\tilde{n} & :=\frac{n}{.1 \mathrm{fm}^{3}}, \\
\tilde{\rho} & :=\rho \cdot \frac{G_{0}^{3} M_{\odot}^{2}}{c^{8}}, \\
\tilde{p} & :=p \cdot \frac{G_{0}^{3} M_{\odot}^{2}}{c^{8}}, \\
\tilde{\phi} & :=\phi \cdot \frac{\sqrt{G_{0}}}{c^{2}}, \\
\tilde{V}(\tilde{\phi}) & :=V(\tilde{\phi}) \cdot \frac{G_{0}^{3} M_{\odot}^{2}}{c^{8}}, \\
\tilde{G}_{\mathrm{eff}} & :=\frac{1}{1+16 \pi \xi \tilde{\phi}^{2}},
\end{aligned}
$$

then the final form of the equations to be solved numerically is :

$$
\begin{aligned}
\partial_{\tilde{r}} \tilde{m}= & 4 \pi \tilde{r}^{2} \tilde{E}, \\
\partial_{\tilde{r}} \tilde{\nu}= & \frac{A^{2}}{1+16 \pi \xi \tilde{r} \phi\left(\partial_{\tilde{r}} \phi\right) \tilde{G}_{\mathrm{eff}}}\left\{\frac{\tilde{m}}{\tilde{r}^{2}}+4 \pi \tilde{r} \tilde{G}_{\mathrm{eff}}\left[\frac{1}{2 A^{2}}\left(\partial_{\tilde{r}} \phi\right)^{2}-\tilde{V}(\phi)+\tilde{p}-\frac{8 \xi \phi \partial_{\tilde{r}} \phi}{\tilde{r} A^{2}}\right]\right\}, \\
\partial_{\tilde{r} \tilde{r}} \phi= & -\left[\frac{2}{\tilde{r}}+\partial_{\tilde{r}} \tilde{\nu}-\left(1-\frac{2 \tilde{m}}{\tilde{r}}\right)^{-1}\left(4 \pi \tilde{r} \tilde{E}-\frac{\tilde{m}}{\tilde{r}^{2}}\right)\right] \partial_{\tilde{r}} \phi \\
& +\left(1-\frac{2 \tilde{m}}{\tilde{r}}\right)^{-1}\left[\frac{\partial \tilde{V}(\phi)}{\partial \phi}-16 \pi \xi \phi(\tilde{E}-\tilde{S})\right], \\
\partial_{\tilde{r}} \tilde{n}= & -\frac{(\tilde{\rho}+\tilde{p}) \tilde{n}}{\gamma \tilde{p}} \partial_{\tilde{r} \tilde{\nu}},
\end{aligned}
$$

where

$$
\begin{aligned}
\tilde{E}-\tilde{S}= & \frac{\tilde{G}_{\mathrm{eff}}}{1+192 \pi \xi^{2} \phi^{2} \tilde{G}_{\mathrm{eff}}}\left[\frac{1}{A^{2}}\left(\partial_{\tilde{r}} \phi\right)^{2}(1+12 \xi)+4 \tilde{V}(\phi)+\tilde{\rho}-3 \tilde{p}+12 \xi \phi \frac{\partial \tilde{V}(\phi)}{\partial \phi}\right], \\
\tilde{E}= & \frac{\tilde{G}_{\mathrm{eff}}}{1+192 \pi \xi^{2} \phi^{2} \tilde{G}_{\mathrm{eff}}}\left[-\frac{4 \xi \phi\left(\partial_{\tilde{r}} \phi\right)\left(\partial_{\tilde{r}} \tilde{\nu}\right)}{A^{2}}\left(1+192 \pi \xi^{2} \phi^{2} \tilde{G}_{\mathrm{eff}}\right)+\frac{1}{2 A^{2}}\left(\partial_{\tilde{r}} \phi\right)^{2}\left(1+8 \xi+64 \pi \xi^{2} \phi^{2} \tilde{G}_{\mathrm{eff}}\right)\right. \\
& \left.+4 \xi \phi \frac{\partial \tilde{V}(\phi)}{\partial \phi}+\tilde{V}(\phi)\left(1-64 \pi \xi^{2} \phi^{2} \tilde{G}_{\mathrm{eff}}\right)+\tilde{\rho}\left(1+128 \pi \xi^{2} \phi^{2} \tilde{G}_{\mathrm{eff}}\right)+192 \pi \xi^{2} \phi^{2} \tilde{G}_{\mathrm{eff}} \tilde{p}\right] .
\end{aligned}
$$

Here $\tilde{E}$ and $\tilde{S}$ are dimensionless as in Eq. (33), and we have dropped-out the tilde over $\phi$.

We note that (41) with (39) generalizes the Volkoff-Oppenheimer equation of hydrostatic equilibrium for the model (9). We also note that the sources of the differential equations contain only first order derivatives of the field variables and are thus suitable for numerical integration with a Runge-Kutta algorithm. 
By subtracting Eq. (42) from (43) we can obtain an effective total pressure $\tilde{S} / 3$ from which we recognize individual contributions, like the pressure terms associated with the scalar field

$$
p_{\phi}=-\frac{1}{6 A^{2}}\left(\partial_{\tilde{r}} \phi\right)^{2}-\tilde{V}(\phi)
$$

and the NMC contributions:

$$
\begin{aligned}
p_{\phi}^{\xi}= & \frac{\tilde{G}_{\text {eff }}}{3\left(1+192 \pi \xi^{2} \phi^{2} \tilde{G}_{\text {eff }}\right)}\left[-\frac{4 \xi \phi\left(\partial_{\tilde{r}} \phi\right)\left(\partial_{\tilde{r}} \tilde{\nu}\right)}{A^{2}}\left(1+192 \pi \xi^{2} \phi^{2} \tilde{G}_{\text {eff }}\right)\right. \\
& \left.+\frac{1}{2 A^{2}}\left(\partial_{\tilde{r}} \phi\right)^{2}\left(-1-16 \xi+64 \pi \xi^{2} \phi^{2} \tilde{G}_{\text {eff }}\right)-8 \xi \phi \frac{\partial \tilde{V}(\phi)}{\partial \phi}-\tilde{V}(\phi)\left(3+64 \pi \xi^{2} \phi^{2} \tilde{G}_{\text {eff }}\right)\right]-p_{\phi}, \\
p_{\text {mat }}^{\xi}= & \frac{\tilde{G}_{\text {eff }}}{3\left(1+192 \pi \xi^{2} \phi^{2} \tilde{G}_{\text {eff }}\right)}\left[128 \pi \xi^{2} \phi^{2} \tilde{G}_{\text {eff }} \tilde{\rho}+3 \tilde{p}\left(1+64 \pi \xi^{2} \phi^{2} \tilde{G}_{\text {eff }}\right)\right]-\tilde{p}
\end{aligned}
$$

For simplicity we will consider hereafter only the case $V(\phi)=0$ although the basic results are expected to remain qualitatively unchanged.

Regarding the equation of state, we make the standard assumption that the nuclear matter in neutron stars is considered to be at zero temperature (cold catalyzed matter) 15. This matter is represented by an equation of state usually parameterized by the baryon density number:

$$
\begin{aligned}
& p=p(n), \\
& \rho=\rho(n) .
\end{aligned}
$$

In general the EOS are not given analytically. In particular, for realistic EOS, i.e., those built from effective field-theories or many-body nuclear calculations, the above set of equations is given in tabulated form. Thus, a preliminary interpolation is required. In this paper we will use the collection of EOS of [16,17] constructed with a logarithmic interpolation. In particular we show results for three EOS representing the different degrees of stiffness typically found in other models. These are the model of Pandharipande in which only neutrons are taken into account [19] (hereafter referred as to PandN; representative of a "soft" EOS), the model II of Díaz-Alonso [18] (hereafter referred as to DiazII; representative of a "medium" EOS), and the model "0.17" of Haensel et al. [20] (herafter referred as to HKP; representative of a "stiff" EOS). We point out that the DiazII and HKP models, being relativistic models, are thus causal in all the domain of baryon densities. The PandN model, despite being non-relativistic, is causal for the range of densities usually found in stable configurations of neutron stars. We refer the reader to the previous references, and the bibliography therein for a more detailed description of such EOS.

\section{BOUNDARY CONDITIONS AND NUMERICAL METHODOLOGY}

Interior solution. The regularity condition at $r=0$ (center of the star) on the metric requires the boundary condition 


$$
m(0)=0
$$

The boundary condition on $\tilde{\nu}(r)$ is by definition [cf.Eq. (19)]

$$
\tilde{\nu}(0) \equiv 0
$$

The integration of Eq. (41) is performed by specifying the baryon density at the center of the star

$$
n(0)=n_{c},
$$

with the regularity condition $\partial_{r} n(0)=0$.

In a similar way the boundary and regularity conditions on the scalar field are

$$
\begin{aligned}
\phi(0) & =\phi_{c}, \\
\partial_{r} \phi(0) & =0 .
\end{aligned}
$$

On the other hand, the value $\phi_{c}$ cannot be arbitrary, but must be so that $\phi$ satisfy the appropriate boundary conditions at spatial infinite. This is enforced by the use of a standard shooting method [21]. In the present paper, we only consider an asymptotically vanishing scalar field. This corresponds to the situation in which spontaneous scalarization might arise. The situation for a non-zero asymptotic value of the scalar field can give rise to "induced" scalarization in the neutron star, and can be also obtained in exactly the same fashion by a shooting method. Figure 1 shows the solution of the Klein-Gordon equation satisfying vanishing asymptotic boundary conditions. Figure 2 shows the "trajectories" $\left(\phi_{c}, \rho_{c}\right)$ for which those conditions are verified within different neutron star configurations.

Exterior solution. We can compute the exterior solution by integrating the equations from the star surface to spatial infinite. Outside the support of the fluid variables $\rho, p$, we "compactify" the space by a transformation on the $r$ coordinate of the kind $u=1 / r$, and integrate the resulting equations (see the Appendix) in the domain $u \in[1 / R, 0]$; at the star radius $R$ (which is obtained numerically) and which is defined as the coordinate $r$ at which the pressure vanishes $p(R)=0$, the values of the field variables are given numerically, and they represent the boundary conditions for the integration of the equations outside the star. The integration constants are fixed by matching continuously the interior with the (numerical calculated) exterior solution.

The physical lapse $N(r)=e^{\nu}$ at $r=0$ is calculated at the end of the numerical integration from $(19)$

$$
\nu(0)=-\tilde{\nu}_{\infty},
$$

where the value $\tilde{\nu}_{\infty}$ is obtained from the numerical integration, and it ensures that at spatial infinite our coordinates correspond to the standard Minkowski coordinates. Figure 3 depicts the metric potentials with asymptotic conditions matching with Minkowski coordinates at spatial infinite.

Global quantities. The staticity of the configurations we are studying ensures that the ADM (gravitational) mass and the Komar mass coincide, and can be easily evaluated from the integral 


$$
M_{\mathrm{ADM}}=\lim _{r \rightarrow \infty} m(r)=4 \pi \int_{0}^{\infty} r^{2} E(r) d r .
$$

We emphasize that the energy density $E$ might not have compact support as it might include contributions of the scalar field. Therefore in order to compute the actual ADM mass the integration have to be performed from the center of the star to spatial infinite.

Moreover, the conservation of the baryon number leads to the conserved total baryon number of the star given by

$$
\mathcal{N}=4 \pi \int_{0}^{R} n(r) A(r) r^{2} d r
$$

The total baryon mass is defined by

$$
M_{\mathrm{bar}}=m_{b} \mathcal{N}
$$

where $m_{b}$ is the mean mass of single baryons $\left(1.66 \times 10^{-27} \mathrm{~kg}\right)$.

As it is usual, we can define the total binding energy of the star as

$$
\mathcal{E}_{\mathrm{bind}}=M_{\mathrm{ADM}}-M_{\mathrm{bar}}
$$

and the fractional binding energy as

$$
\mathcal{E}_{\text {frac }}=1-\frac{M_{\mathrm{bar}}}{M_{\mathrm{ADM}}}
$$

From the asymptotic behavior of the scalar field with vanishing boundary conditions,

$$
\phi(r) \sim \frac{G_{0} \omega}{c^{2} r}+\mathcal{O}\left(1 / r^{2}\right)
$$

one can define the so called "scalar charge" $\omega$ as follows:

$$
\omega:=-\lim _{r \rightarrow \infty}\left[r^{2} \frac{c^{2}}{G_{0}} \frac{d \phi}{d r}\right] .
$$

Following [2,3], we introduce the coupling strength

$$
\alpha:=-\frac{\omega}{M_{\mathrm{ADM}}}
$$

In the next section we provide the numerical analysis of the above quantities, and their dependence on the parameters $\rho_{c}=\rho\left(n_{c}\right), \phi_{c}$, and the equation of state. 


\section{NUMERICAL RESULTS}

\section{A. Relativistic energetic analysis of spontaneous scalarization}

In section III we showed that a naive Newtonian analysis leads to the conclusion that the theory described by (9) with $\xi>0$ should not give rise to a spontaneous scalarization since the presence of the scalar field would tend to increase the total energy by decreasing of the negative binding energy and thus to a configuration with a larger total energy than the corresponding case in absence of scalar field, for a fixed baryonic mass. In this section we will show that a numerical analysis of the full relativistic energy contributions confirms that the phenomenon of spontaneous scalarization indeed occurs and that the naive analysis fails.

Let us separate the total energy density $E$ [see Eq. (43)] into contributions that can be identified as representing the different effects that arise in the theory. This separation of course can be consider to have no more than heuristic value, for even the energy density itself has no invariant meaning in a diffeomorphism invariant theory. We do this separation in the following way

$$
\begin{aligned}
& \rho_{\text {int }}(r):==m_{b} n(r), \\
& \rho_{\mathrm{bar}}^{\text {eff }}(r):=m_{b} n(r) A(r) \\
& \rho_{\mathrm{bar}}^{\mathrm{bind}}(r):= \rho_{\mathrm{bar}}^{\mathrm{eff}}(r)-m_{b} n(r), \\
& E_{\phi}:= \frac{1}{2 A^{2}}\left(\partial_{r} \phi\right)^{2}, \\
& E_{\phi}^{\xi}:= \frac{G_{\mathrm{eff}}}{1+192 \pi \xi^{2} \phi^{2} G_{\mathrm{eff}}}\left[-\frac{4 \xi \phi\left(\partial_{r} \phi\right)\left(\partial_{r} \tilde{\nu}\right)}{A^{2}}\left(1+192 \pi \xi^{2} \phi^{2} G_{\mathrm{eff}}\right)\right. \\
&\left.+\frac{1}{2 A^{2}}\left(\partial_{r} \phi\right)^{2}\left(1+8 \xi+64 \pi \xi^{2} \phi^{2} G_{\mathrm{eff}}\right)\right]-E_{\phi}, \\
& E_{\rho}^{\xi}:= \frac{G_{\mathrm{eff}}}{1+192 \pi \xi^{2} \phi^{2} G_{\mathrm{eff}}}\left[\rho\left(1+128 \pi \xi^{2} \phi^{2} G_{\mathrm{eff}}\right)+192 \pi \xi^{2} \phi^{2} G_{\mathrm{eff}} p\right]-\rho .
\end{aligned}
$$

Then it is clear that

$$
E=\rho_{\mathrm{bar}}^{\mathrm{eff}}-\rho_{\mathrm{bar}}^{\mathrm{bind}}+\rho_{\mathrm{int}}+E_{\phi}+E_{\phi}^{\xi}+E_{\rho}^{\xi} .
$$

This decomposition has the following interpretation: $\rho_{\mathrm{bar}}^{\mathrm{eff}}$ is the physical baryon density, that is, the energy density that will give rise to the total baryon mass; $\rho_{\text {int }}$ is the interaction energy between baryons (usually it is given in a complicated way related to the physics model of the nuclear matter), $-\rho_{\mathrm{bar}}^{\mathrm{bind}}$ is the negative standard binding energydensity of baryons (is the part that is associated only with the difference of the baryon density multiplied by the geometrical factor corresponding to the proper volume element on spacelike hypersurfaces $\Sigma_{t}$ and the baryon density itself. The integral of this difference is what yields the total binding baryon energy in the case of pure general relativity [22]), $E_{\phi}$ is the energy-density of the scalar field as it is usually defined without NMC, $E_{\phi}^{\xi}$ is the contribution to the energy-density of the scalar field due to the non-minimimal coupling 
(note that this vanishes when $\xi=0$ ), and finally, $E_{\rho}^{\xi}$ is the contribution to the total matter-energy density due to the presence of the non-minimal coupling (we also note that it vanishes for $\xi=0$ ). The integration of each of the terms from $r=0$ to $r=+\infty$ will give the total energy contributions:

$$
M_{\mathrm{ADM}}=M_{\mathrm{bar}}+M_{\mathrm{int}}-M_{\mathrm{bind}}+M_{\phi}+M_{\phi}^{\xi}+M_{\rho}^{\xi}
$$

We remark that the above interpretation is sustainable as far as we concern ourselves with the functional form of the terms themselves and regard them as applicable to different test configurations. On the other hand the particular configuration that minimizes the total $M_{\mathrm{ADM}}$ arises from an interplay of all such effects and therefore the actual value of each of the terms in Eq. (70) is affected by the presence of the other terms. Nevertheless the heuristic value of the arguments is validated since such affectations can be consider as higher order effects.

In the absence of scalar field we have

$$
M_{\mathrm{ADM}}^{\mathrm{GR}}=M_{\mathrm{bar}}^{\mathrm{GR}}+M_{\mathrm{int}}^{\mathrm{GR}}-M_{\mathrm{bind}}^{\mathrm{GR}}
$$

So if we want to compare two configurations at fixed baryon mass $\left(M_{\mathrm{bar}}=M_{\mathrm{bar}}^{\mathrm{GR}}\right)$, one with $\phi \neq 0$ and the other with null scalar field, we have

$$
\begin{aligned}
\Delta M & :=M_{\mathrm{ADM}}-M_{\mathrm{ADM}}^{\mathrm{GR}} \\
& =M_{\mathrm{int}}-M_{\mathrm{int}}^{\mathrm{GR}}-\left(M_{\mathrm{bind}}-M_{\mathrm{bind}}^{\mathrm{GR}}\right)+M_{\phi}+M_{\phi}^{\xi}+M_{\rho}^{\xi} .
\end{aligned}
$$

Now according to the Newtonian intuition we expect that $\left|M_{\text {bind }}^{\mathrm{GR}}\right|>\left|M_{\text {bind }}\right|$. So the term $-\left(M_{\text {bind }}-M_{\text {bind }}^{\mathrm{GR}}\right)$ would be positive. Moreover the energy $M_{\phi}$ is also positive and we expect that $M_{\mathrm{int}}-M_{\mathrm{int}}^{\mathrm{GR}}$ is small. In order to have spontaneous scalarization we need $\Delta M<0$ and so the term $M_{\phi}^{\xi}+M_{\rho}^{\xi}$ should be negative enough to more than compensate for the positive energy contributions and also for the decrease in the ordinary binding energy. Figure 1 shows the local behavior of such energy contributions (referred with lower-case letters in the caption) for a configuration with spontaneous scalarization, and confirms the previous conjectures. Figure 5 depicts the corresponding energy-densities as defined by the Eqs. (63)- 68]). Table I shows examples of configurations exhibiting spontaneous scalarization as compared with those in absence of scalar field at the same baryon mass. We can appreciate from table I that the different energy contributions add to reproduce the ADM mass and that the value for the configuration with $\phi \neq 0$ is lower than its corresponding value with $\phi=0$. Note also that the binding baryon energies in absence of scalar field are smaller than their corresponding in the configurations with $\phi \neq 0$. This confirms the original Newtonian intuition. However, the relativistic energetic analysis also shows that the Newtonian naive argument failed to explain the spontaneous scalarization with $\xi>0$ since it did not take into account the large negative energy contribution $M_{\rho}^{\xi}$ which is the responsible for the decrease of the total energy $\left(M_{\phi}^{\xi}\right.$ turns to be always positive), and as it is shown in the examples of table I, is the dominant part of the above difference $\Delta M$. This contribution as well as $M_{\phi}^{\xi}$ have no Newtonian counterpart. 


\section{B. Effects of the equations of state}

A simple and enlightening way to present the effects of the equation of state on the phenomena behavior of the other global quantities as a function of the total baryon mass of the neutron star. Figures 6 and 7 show the behavior of the coupling strength $\alpha$ [see Eq. (62 as a function of the total baryon mass for three EOS. We note that there are critical values $M_{\mathrm{bar}}^{\text {crit }}$ beyond which the phenomenon of spontaneous scalarization ensues. The critical values correspond to the point at which configurations with a non-vanishing scalar field become energetically more favorable than the configurations with $\phi(r)=0$. This behavior can be better appreciated in Fig. 8. It is interesting to note that $M_{\text {bar }}^{\text {crit }}$ is an increasing function of the stiffness of the EOS (for example when $\xi=2, M_{\mathrm{bar}}^{\text {crit }}$ for the HKP EOS increases in $\sim 37 \%$ relative to that for the PandN EOS.), but that the maximum values of $\alpha$ seem to be almost independent of the EOS.

Figure 7 shows that the larger the value of $\xi$ the less important the effect of the EOS on the critical value $M_{\mathrm{bar}}^{\text {crit }}$. This can be understood on basic grounds by looking at the expression of $E_{\rho}^{\xi}$ [Eq. (68)], which as we discussed is the main responsible for spontaneous scalarization. As $\xi$ increases $E_{\phi}^{\xi} \rightarrow-\rho$. This means that its relative contribution is becoming dominant regardless of the central-value of $\rho$. Therefore spontaneous scalarization can take place for smaller values of $\rho_{c}$. Furthermore, the lower the value $\rho_{c}$ the more alike are the equations of state since the three EOS are built with the same standard neutronstar-crust EOS which corresponds to that of [23]. So the configurations of the star with lower central values of $\rho$ are very much alike. This is the reason for the critical baryon

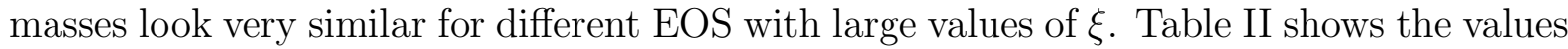
of quantities corresponding to the onset of spontaneous scalarization (i.e., corresponding to the critical $M_{\text {bar }}$ ).

As it was pointed out in [3], the non-perturbative effects of the scalar field in the neutron star are the analogue of spontaneous magnetization in ferromagnets at temperatures lower than the Curie point. In the Landau ansatz where the free energy $\Gamma$ of the ferromagnet is a function of the magnetization $\mu$ in the form $\Gamma=a\left(T-T_{C}\right) \mu^{2} / 2+b \mu^{4} / 4$, it appears two non-trivial minima for temperatures $T<T_{C}$. The analogue to the curve giving the locus of the minima of $\Gamma$, is depicted for the case of spontaneous scalarization in figure 9, where the scalar charge $\omega$ plays the role of $\mu$, the total mass $M_{\mathrm{ADM}}$ the role of $\Gamma$, and finally the baryon mass is the analogue of the temperature. Obviously in this case, the complexity of the system makes difficult an attempt to provide an analytic expression of the form $M_{\mathrm{ADM}}=M_{\mathrm{ADM}}\left(\omega, M_{\mathrm{bar}}\right)$ for which we could recover the behavior of figure 9 .

Another effect related to the phenomenon of spontaneous scalarization is the increase of the maximum masses (both $M_{\mathrm{ADM}}$ and $M_{\mathrm{bar}}$ ) as compared with the values allowed by general relativity. Figure 10 shows the dependence of $M_{\mathrm{ADM}}$ on $\rho_{c}$ for the selected EOS. The behavior of these profiles are qualitatively the same as in general relativity (see Ref. [16]). Table III gives the relevant properties of the neutron star models corresponding to the maximum mass configurations. Note the increase of $M_{\mathrm{ADM}}$ with $\xi$. 


\section{DISCUSSION}

One of the main goals of this work was to understand the discrepancy between the Newtonian expectations and the relativistic energetic analysis. A fortiori it turns out that the difference can be understood by noting the different roles that the gravitational constant plays in the energy considerations in the Newtonian and the relativistic theories. As discussed in section III, the Newtonian point of view leads to the expectation that the total mass should be expressed in the form:

$$
\mathcal{M}=\int \rho_{\mathrm{bar}}(\bar{x}) d^{3} \bar{x}+\int \rho_{\phi}(\bar{x}) d^{3} \bar{x}-G_{\mathrm{eff}}(\phi) \int \frac{\rho_{\mathrm{bar}}(\bar{x}) \rho_{\mathrm{bar}}(\bar{y})}{|\bar{x}-\bar{y}|} d^{3} \bar{x} d^{3} \bar{y}+\ldots
$$

so that the gravitational constant appears as the coefficient of a term that represents the binding energy which is negative, so a decrease in $G_{\text {eff }}$ results in an increase on $\mathcal{M}$. In the relativistic theory the ADM mass itself is of gravitational origin because it is a measure of the departure of the metric (at large distances) from the Minkowski metric, i.e., it represents the $1 / r$ coefficient of such deviation. In this sense we can say that the only thing which has meaning is the combination $G \times \mathcal{M}$ and not $\mathcal{M}$ itself. Thus the quantity that must be minimized is (heuristically):

$$
G \mathcal{M}=G_{\text {eff }}(\phi)\left[\int \rho_{\text {bar }}(\bar{x}) d^{3} \bar{x}+\int \rho_{\phi}(\bar{x}) d^{3} \bar{x}-G_{\text {eff }}(\phi) \int \frac{\rho_{\text {bar }}(\bar{x}) \rho_{\text {bar }}(\bar{y})}{|\bar{x}-\bar{y}|} d^{3} \bar{x} d^{3} \bar{y}+\ldots \quad,\right]
$$

Which we can write as

$$
G \mathcal{M}=G_{\mathrm{eff}}(\phi) \mathcal{M}_{\mathrm{bar}}+G_{\mathrm{eff}}(\phi) \mathcal{M}_{\phi}-G_{\mathrm{eff}}^{2}(\phi) \int \frac{\rho_{\mathrm{bar}}(\bar{x}) \rho_{\mathrm{bar}}(\bar{y})}{|\bar{x}-\bar{y}|} d^{3} \bar{x} d^{3} \bar{y}+\ldots,
$$

The point is that the appearance of a nonzero value of $\phi$ which results in the second term becoming positive (from a zero value when $\phi=0$ ) can be more than compensated by the decrease in the first term due to the decrease in $G_{\text {eff }}$ that is associated with an increase in $\phi$. The change in the third term, which in effect is in the direction inferred from the Newtonian analysis, playing now a secondary role. This explains why the Newtonian analysis failed and why the relativistic analysis shows that the phenomenon of spontaneous scalarization is associated with an effective gravitational constant that decreases with $\phi$. This explanation is supported by the numerical analysis since the term $M_{\rho}^{\xi}$ which represents precisely the effect described here turns out to be the one that is responsible for the lowering of the ADM mass of the configuration with a scalar field in comparison to the configuration without one.

The second goal of this paper was to study the dependence of the parameters associated with the spontaneous scalarization with the equation of state of the nuclear matter. This can be summarized as follows. First the critical baryon masses at which spontaneous scalarization develops increases as the equation of state becomes more rigid. As $\xi$ increases those critical values become much less dependent of the EOS. Second, the maximum values of the coupling strength $\alpha$ seem to be independent of the EOS. Finally, the effect of the scalar field makes the neutron star to support larger masses than in general relativity, and this is more evident as $\xi$ increases. 


\section{ACKNOWLEDGMENTS}

We wish to thank H. Quevedo for helpful discussions. M.S and D.S. would like to acknowledge partial support from DGAPA-UNAM project IN105496 and to thank the supercomputing department of DGSCA-UNAM. 


\section{APPENDIX: DIMENSIONLESS FORM OF FIELD EQUATIONS AT THE EXTERIOR}

By performing a "conformal" transformation of the kind $u=1 / \tilde{r}$ in Eqs. (38)-(43) (with $\tilde{\rho}=\tilde{p}=V(\phi)=0$ ) we obtain the equations to be solved in the domain $u \in[1 / R, 0]$ which corresponds to the range from the radius of the star to spatial infinite.

$$
\begin{aligned}
\partial_{u} \tilde{m}= & -4 \pi \tilde{E}_{u}, \\
\partial_{u} \tilde{\nu}= & -\frac{(1-2 \tilde{m} u)^{-1}}{1-16 \pi \xi u \phi\left(\partial_{u} \phi\right) \tilde{G}_{\mathrm{eff}}}\left\{\tilde{m}+4 \pi \tilde{G}_{\mathrm{eff}}(1-2 \tilde{m} u)\left[\frac{u}{2}\left(\partial_{u} \phi\right)^{2}+8 \xi \phi \partial_{u} \phi\right]\right\}, \\
\partial_{u u} \phi= & -\left[\partial_{u} \tilde{\nu}-(1-2 \tilde{m} u)^{-1}\left(\tilde{m}-4 \pi u \tilde{E}_{u}\right)\right] \partial_{u} \phi \\
& -\frac{16 \pi \xi \phi \tilde{G}_{\mathrm{eff}}}{1+192 \pi \xi^{2} \phi^{2} \tilde{G}_{\mathrm{eff}}}\left(\partial_{u} \phi\right)^{2}(1+12 \xi), \\
\tilde{E}_{u}= & \frac{\tilde{G}_{\mathrm{eff}}(1-2 \tilde{m} u)}{1+192 \pi \xi^{2} \phi^{2} \tilde{G}_{\mathrm{eff}}}\left[-4 \xi \phi\left(\partial_{u} \phi\right)\left(\partial_{u} \tilde{\nu}\right)\left(1+192 \pi \xi^{2} \phi^{2} \tilde{G}_{\mathrm{eff}}\right)\right. \\
& \left.+\frac{1}{2}\left(\partial_{u} \phi\right)^{2}\left(1+8 \xi+64 \pi \xi^{2} \phi^{2} \tilde{G}_{\mathrm{eff}}\right)\right]
\end{aligned}
$$




\section{TABLES}

TABLE I. Examples of configurations exhibiting spontaneous scalarization compared with those at the same baryon mass in absence of scalar field. $\xi$ is the non-minimal coupling constant; $M_{\text {bar }}$ is the total baryon mass; $M_{\text {bin }}$ is the baryon-binding energy; $M_{\text {int }}$ is the total baryon-interaction energy; $M_{\phi}$ is the total mass contribution due to the single scalar field; $M_{\rho}^{\xi}$ is the mass contribution of the correction to the total matter-energy by the presence of the non-minimal coupling; $M_{\phi}^{\xi}$ is the mass contribution of the correction to $M_{\phi}$ by the presence of the non-minimal coupling; $M_{\mathrm{ADM}}$ is the ADM-mass. We use $M_{\odot}=1.989 \times 10^{30} \mathrm{~kg}$.

\begin{tabular}{c|ccccccccc}
\hline \hline EOS & $\xi$ & $\phi(r)$ & $\begin{array}{c}M_{\text {bar }} \\
{\left[M_{\odot}\right]}\end{array}$ & $\begin{array}{c}M_{\text {bind }} \\
{\left[M_{\odot}\right]}\end{array}$ & $\begin{array}{c}M_{\text {int }} \\
{\left[M_{\odot}\right]}\end{array}$ & $\begin{array}{c}M_{\phi} \\
{\left[M_{\odot}\right]}\end{array}$ & $\begin{array}{c}M_{\rho}^{\xi} \\
{\left[M_{\odot}\right]}\end{array}$ & $\begin{array}{c}M_{\phi}^{\xi} \\
{\left[M_{\odot}\right]}\end{array}$ & $\begin{array}{c}M_{\text {ADM }} \\
{\left[M_{\odot}\right]}\end{array}$ \\
\hline DiazII & 2 & $\neq 0$ & 1.563 & -0.179 & 0.082 & 0.011 & -0.226 & 0.179 & 1.429 \\
& 2 & $=0$ & 1.563 & -0.207 & 0.077 & 0 & 0 & 0 & 1.433 \\
& 6 & $\neq 0$ & 2.170 & -0.320 & 0.170 & 0.011 & -0.559 & 0.413 & 1.885 \\
& 6 & $=0$ & 2.170 & -0.469 & 0.204 & 0 & 0 & 0 & 1.904 \\
\hline \hline
\end{tabular}

TABLE II. Critical configurations towards spontaneous scalarization. $n_{\mathrm{c}}, \rho_{\mathrm{c}}$ are the central baryon and proper energy densities respectively, $p_{\mathrm{c}}$ is the central pressure, $R$ is the radius of the star, $N_{\mathrm{c}}$ is the value of the lapse at the center of the star. The remaining symbols are defined in the caption of Table 1 . We use $G_{0}=6.672 \times 10^{-11} \mathrm{~m}^{3} \mathrm{~kg}^{-1} \mathrm{~s}^{-2}, c=2.9979 \times 10^{8} \mathrm{~m} \mathrm{~s}^{-1}$, $M_{\odot}=1.989 \times 10^{30} \mathrm{~kg}$, and $\rho_{\text {nuc }}=1.66 \times 10^{17} \mathrm{~kg} \mathrm{~m}^{-3}$.

\begin{tabular}{c|cccccccccc}
\hline \hline EOS & $\xi$ & $\begin{array}{c}n_{\mathrm{c}} \\
{\left[.1 \mathrm{fm}^{-3}\right]}\end{array}$ & $\begin{array}{c}\rho_{\mathrm{c}} \\
{\left[\rho_{\mathrm{nuc}} c^{2}\right]}\end{array}$ & $\begin{array}{c}p_{\mathrm{c}} \\
{\left[\rho_{\mathrm{nuc}} c^{2}\right]}\end{array}$ & $\begin{array}{c}M_{\mathrm{ADM}} \\
{\left[M_{\odot}\right]}\end{array}$ & $\begin{array}{c}M_{\mathrm{bar}} \\
{\left[M_{\odot}\right]}\end{array}$ & $\begin{array}{c}R \\
{[\mathrm{~km}]}\end{array}$ & $1-\frac{M_{\mathrm{bar}}}{M_{\mathrm{ADM}}}$ & $\frac{2 G_{0} M_{\mathrm{ADM}}}{c^{2} R}$ & $N_{c}$ \\
\hline PandN & 2 & 4.22 & 4.46 & 0.254 & 0.596 & 0.617 & 11.29 & -0.035 & 0.156 & 0.822 \\
& 6 & 1.76 & 1.80 & 0.026 & 0.1779 & 0.1779 & 18.65 & $-5.87 \times 10^{-5}$ & 0.028 & 0.944 \\
\hline DiazII & 2 & 2.52 & 2.65 & 0.150 & 0.757 & 0.787 & 13.90 & -0.038 & 0.160 & 0.824 \\
& 6 & 1.17 & 1.20 & 0.018 & 0.2077 & 0.2079 & 20.08 & -0.0012 & 0.030 & 0.945 \\
\hline HKP & 2 & 1.97 & 2.05 & 0.122 & 0.811 & 0.845 & 14.24 & -0.042 & 0.169 & 0.826 \\
& 6 & 1.243 & 1.27 & 0.020 & 0.1970 & 0.1973 & 18.98 & -0.0012 & 0.030 & 0.945 \\
\hline \hline
\end{tabular}


TABLE III. Maximum mass models. $\phi_{\mathrm{c}}$ is the central scalar field, $M_{\mathrm{s}}$ is the "gravitational mass" at the surface of the star, $\omega$ is the scalar charge, $\alpha$ is the coupling strength between the scalar field and gravitation. The remaining identifiers are defined in the caption of Tables [ 【1. The configurations in absence of scalar field corresponds to the maximum mass models in general relativity.

\begin{tabular}{|c|c|c|c|c|c|c|c|c|c|c|c|c|c|c|}
\hline EOS & $\xi$ & $\begin{array}{c}n_{\mathrm{c}} \\
{\left[.1 \mathrm{fm}^{-3}\right]}\end{array}$ & $\begin{array}{c}\rho_{\mathrm{c}} \\
{\left[\rho_{\mathrm{nuc}} c^{2}\right]}\end{array}$ & $\begin{array}{c}p_{\mathrm{c}} \\
{\left[\rho_{\text {nuc }} c^{2}\right]}\end{array}$ & $\phi_{\mathrm{c}}$ & $\begin{array}{c}M_{\mathrm{S}} \\
{\left[M_{\odot}\right]}\end{array}$ & $\begin{array}{c}M_{\mathrm{ADM}} \\
{\left[M_{\odot}\right]}\end{array}$ & $\begin{array}{l}M_{\text {bar }} \\
{\left[M_{\odot}\right]}\end{array}$ & $\begin{array}{c}R \\
{[\mathrm{~km}]}\end{array}$ & $\begin{array}{c}\omega \\
{\left[M_{\odot}\right]}\end{array}$ & $1-\frac{M_{\mathrm{bar}}}{M_{\mathrm{ADM}}}$ & $\frac{2 G_{0} M_{\mathrm{ADM}}}{c^{2} R}$ & $\alpha$ & $N_{c}$ \\
\hline \multirow[t]{3}{*}{ PandN } & 0 & 17.36 & 24.69 & 11.69 & 0 & 1.662 & 1.662 & 1.932 & 8.52 & 0 & -0.162 & 0.575 & 0 & 0.310 \\
\hline & 2 & 15.51 & 20.98 & 8.73 & 0.0171 & 1.620 & 1.694 & 1.981 & 8.91 & 0.0536 & -0.169 & 0.558 & -0.0316 & 0.344 \\
\hline & 6 & 15.51 & 20.98 & 8.73 & 0.0169 & 1.570 & 1.776 & 2.118 & 9.13 & 0.0830 & -0.192 & 0.574 & -0.0467 & 0.337 \\
\hline \multirow[t]{3}{*}{ DiazII } & 0 & 11.14 & 1 & 5.30 & 0 & 1.933 & 1.933 & 2.210 & 10.92 & 0 & -0.143 & 0.522 & 0 & 0.375 \\
\hline & 2 & 10.75 & 14.49 & 4.90 & 0.0226 & 1.868 & 1.990 & 2.297 & 11.21 & 0.0818 & -0.153 & 0.524 & -0.0411 & 0.380 \\
\hline & 6 & 10.94 & 14.85 & 5.10 & 0.019 & 1.801 & 2.085 & 2.451 & 11.42 & 0.115 & -0.175 & 0.538 & -0.055 & 0.368 \\
\hline \multirow[t]{3}{*}{ HKP } & 0 & 6.32 & 8.76 & 4.29 & 0 & 2.835 & 2.835 & 3.421 & 13.67 & 0 & -0.206 & 0.612 & 0 & 0.301 \\
\hline & 2 & 5.64 & 7.42 & 3.27 & 0.0146 & 2.768 & 2.879 & 3.492 & 14.15 & 0.0805 & -0.212 & 0.600 & -0.0279 & 0.332 \\
\hline & 6 & 5.56 & 7.28 & 3.16 & 0.015 & 2.667 & 3.018 & 3.732 & 14.56 & 0.132 & -0.236 & 0.612 & -0.043 & 0.32 \\
\hline
\end{tabular}




\section{FIGURES}

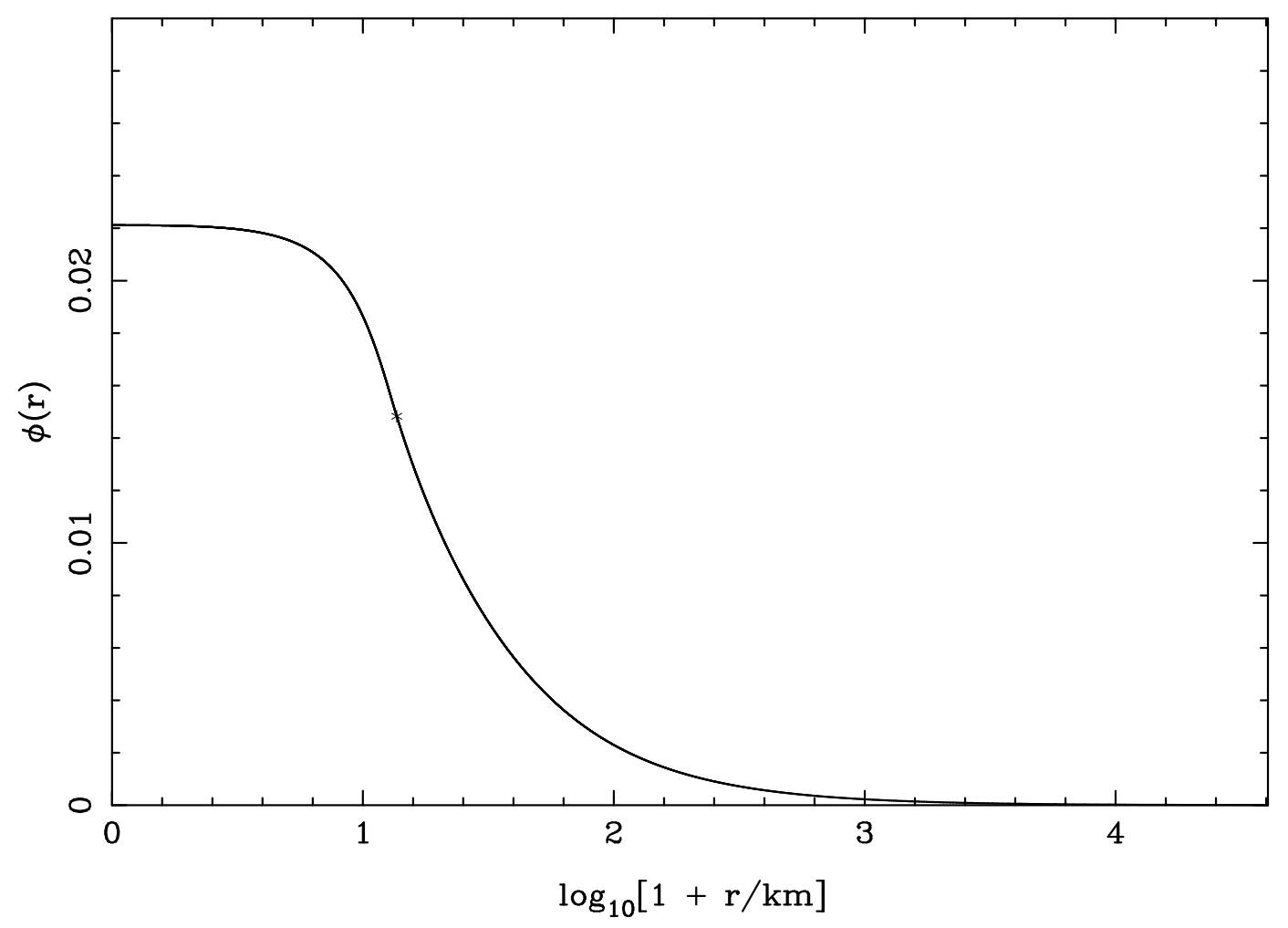

FIG. 1. Scalar-field as a function of $r$ for $\xi=6, n_{\mathrm{c}} \sim 0.7 \mathrm{fm}^{-3}\left(\rho_{\mathrm{c}} \sim 8.37 \rho_{\text {nuc }} c^{2}\right)$ and $\phi_{\mathrm{c}} \sim 0.0221$ for the EOS DiazII. The asterisk depicts the location of the star surface $(R \sim 12.63$ $\mathrm{km})$. The computations includes the domain from $r=0$ to $r=+\infty$, however for pictorial convenience here the logarithmic scale shows the plot only to some extent which includes various orders of magnitude beyond the star surface. 


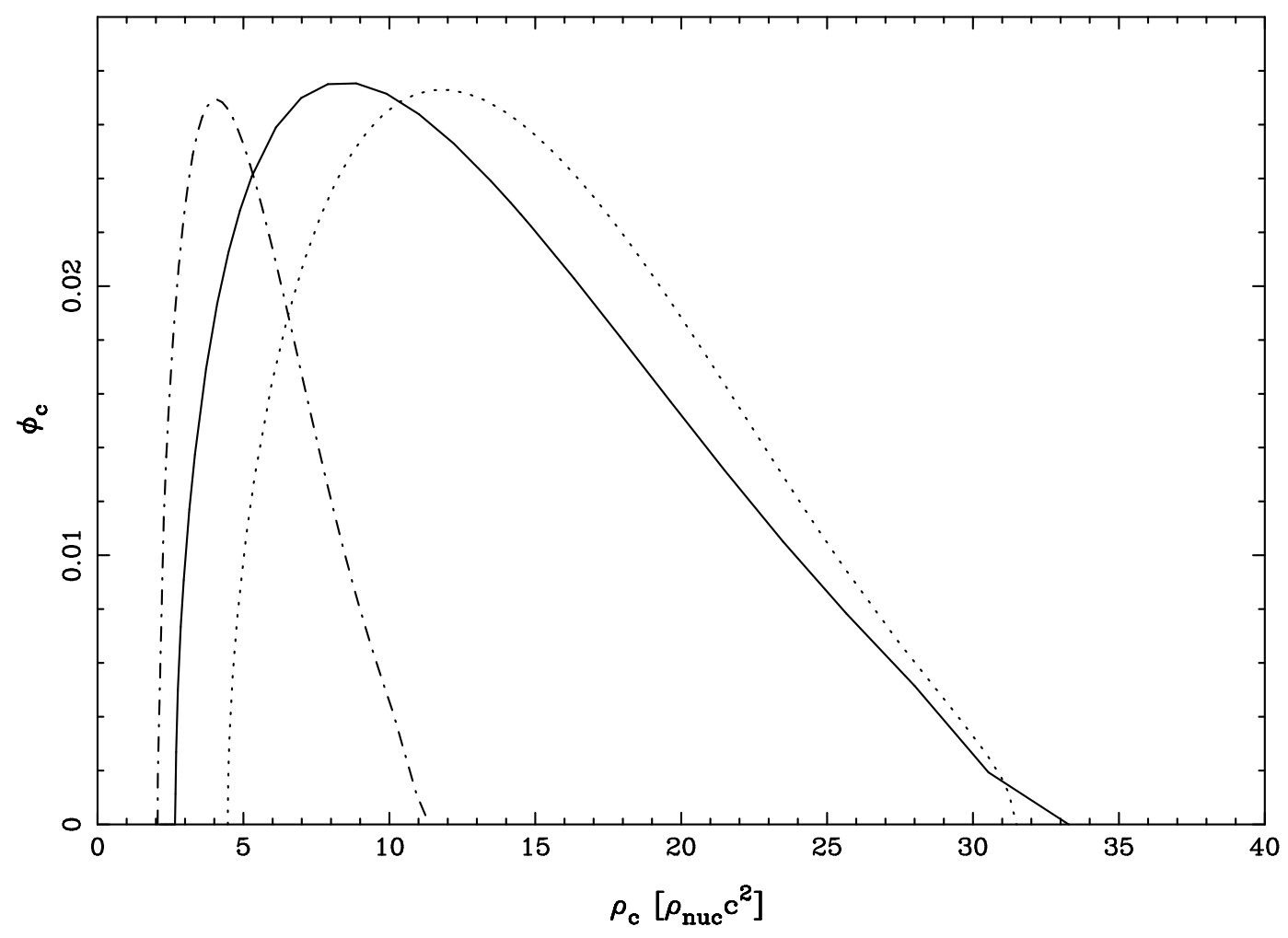

FIG. 2. Functional dependence of $\phi_{c}$ vs $\rho_{c}$ obtained from a shooting method with $\xi=2$ for three different equations of state: HKP (dash-doted line), DiazII (solid line) and PandN (dotted line). The appearence of the non-trivial solutions $\left(\phi_{c} \neq 0\right)$ marks the onset of spontaneous scalarization. 


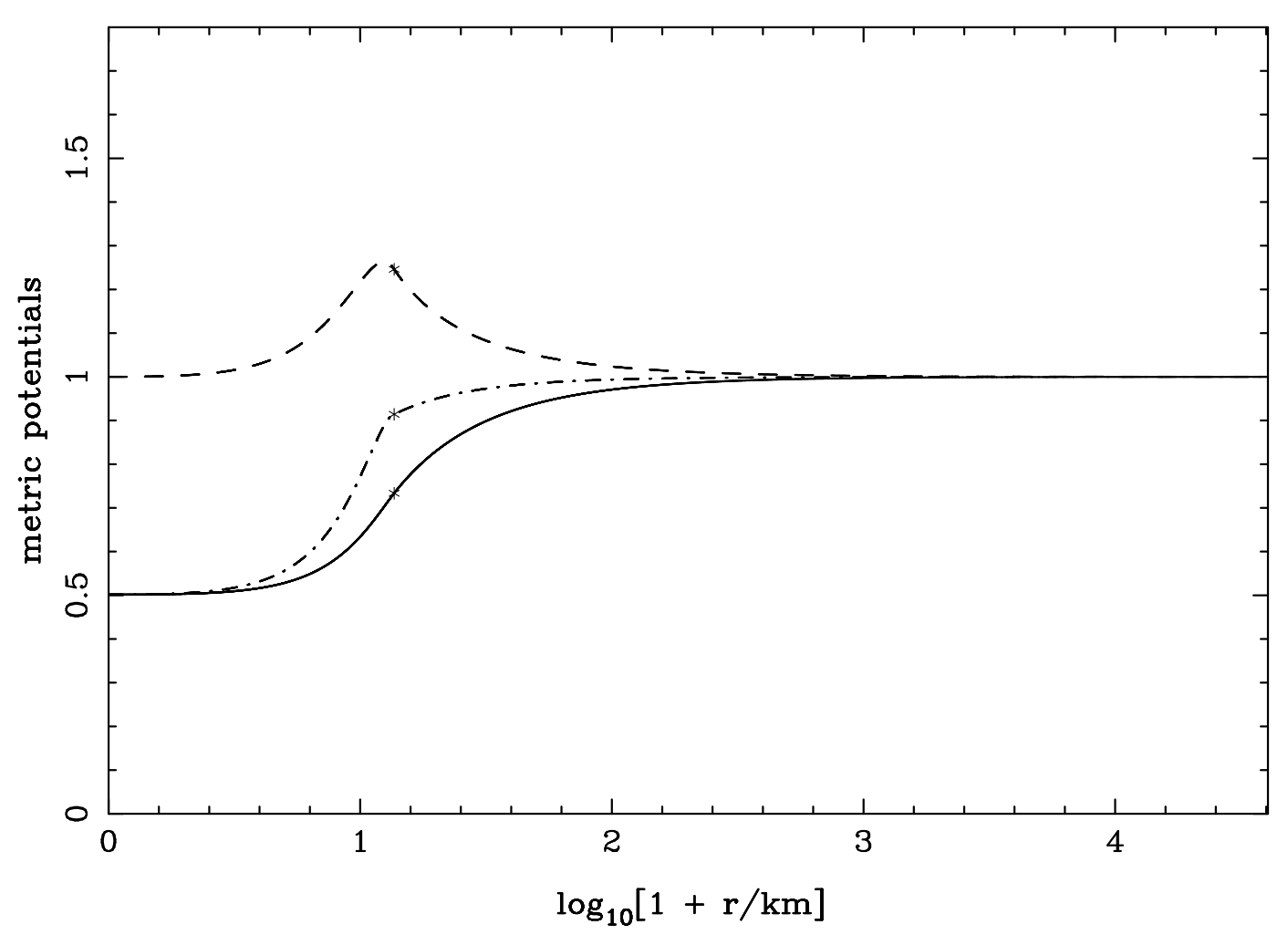

FIG. 3. Metric potentials as a function of $r$ corresponding to the case of Fig. 1. The curves refer to the lapse function $N(r)$ (solid line), to the metric potential $A(r)$ defined by Eq. (20) (dashed line), and to the product $A N$ (dash-dotted line). Note that unlike the Schwarzschild exterior solution this product differs from unit outside the star. Here the asterisks indicates the location of the star surface. 


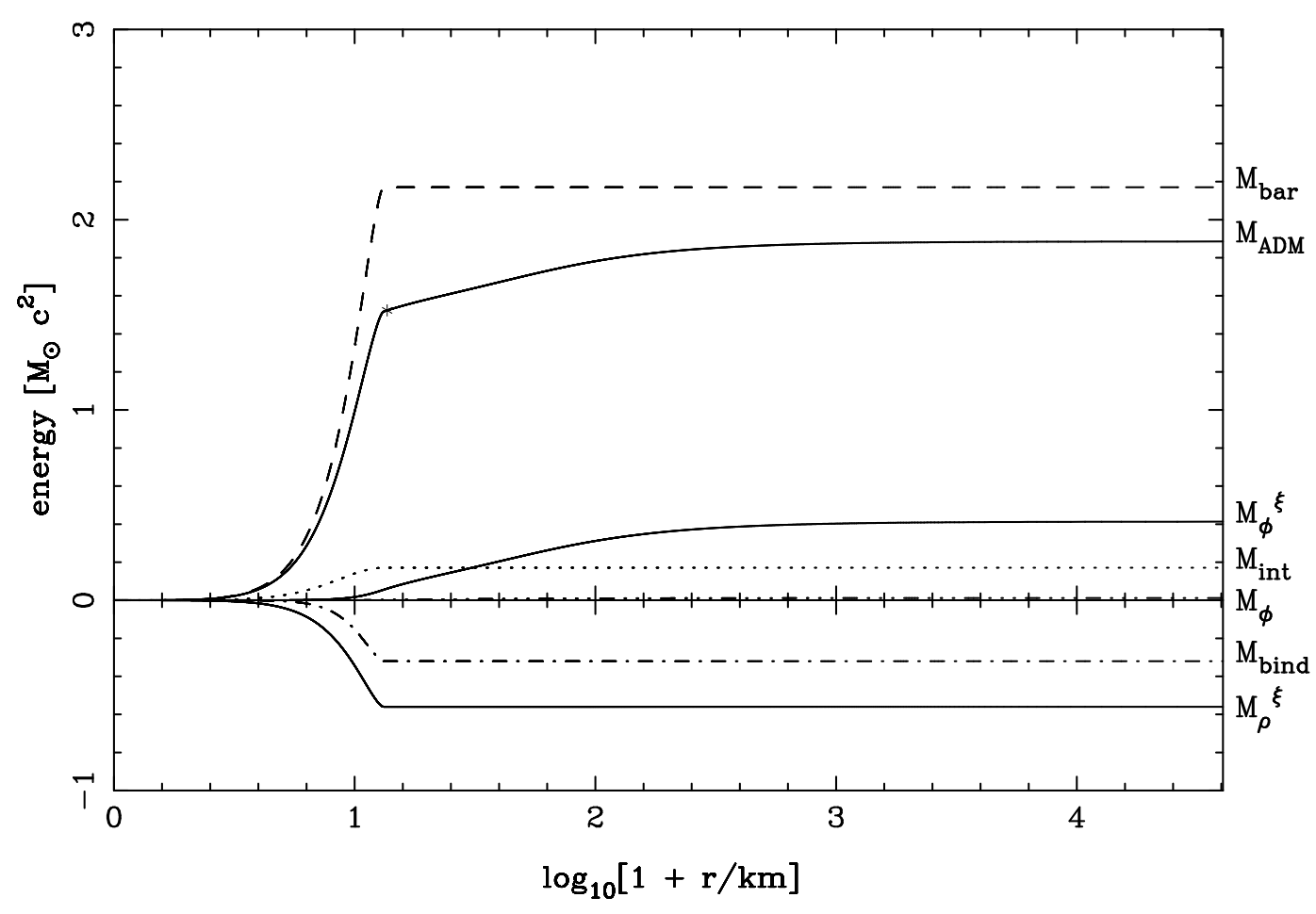

FIG. 4. Energies for the same case of Fig. 11 as a function of $r$. The profiles represent the integration of the energy-densities of Fig. 5 from $r=0$ to $\infty$. The upper solid lines (positive values) correspond to $m(r)$ (larger values) and $m_{\phi}^{\xi}(r)$ (smaller values) respectively; the dashed line stands for $m_{\mathrm{bar}}(r)$; the dotted line corresponds to $m_{\text {int }}(r)$; the dash-dotted line (positive values) corresponds to $m_{\phi}(r)$; the dash-dotted line (negative values) stands for $m_{\text {bind }}(r)$; the lower solid line (negative values) refers to $m_{\rho}^{\xi}(r)$. The asymptotic values of these profiles correspond to those of table 1 . Note that the mass $m(r)$ continues to grow outside the star (the asterisk depicts the star surface) due to the contribution of the energies associated with the scalar field. 


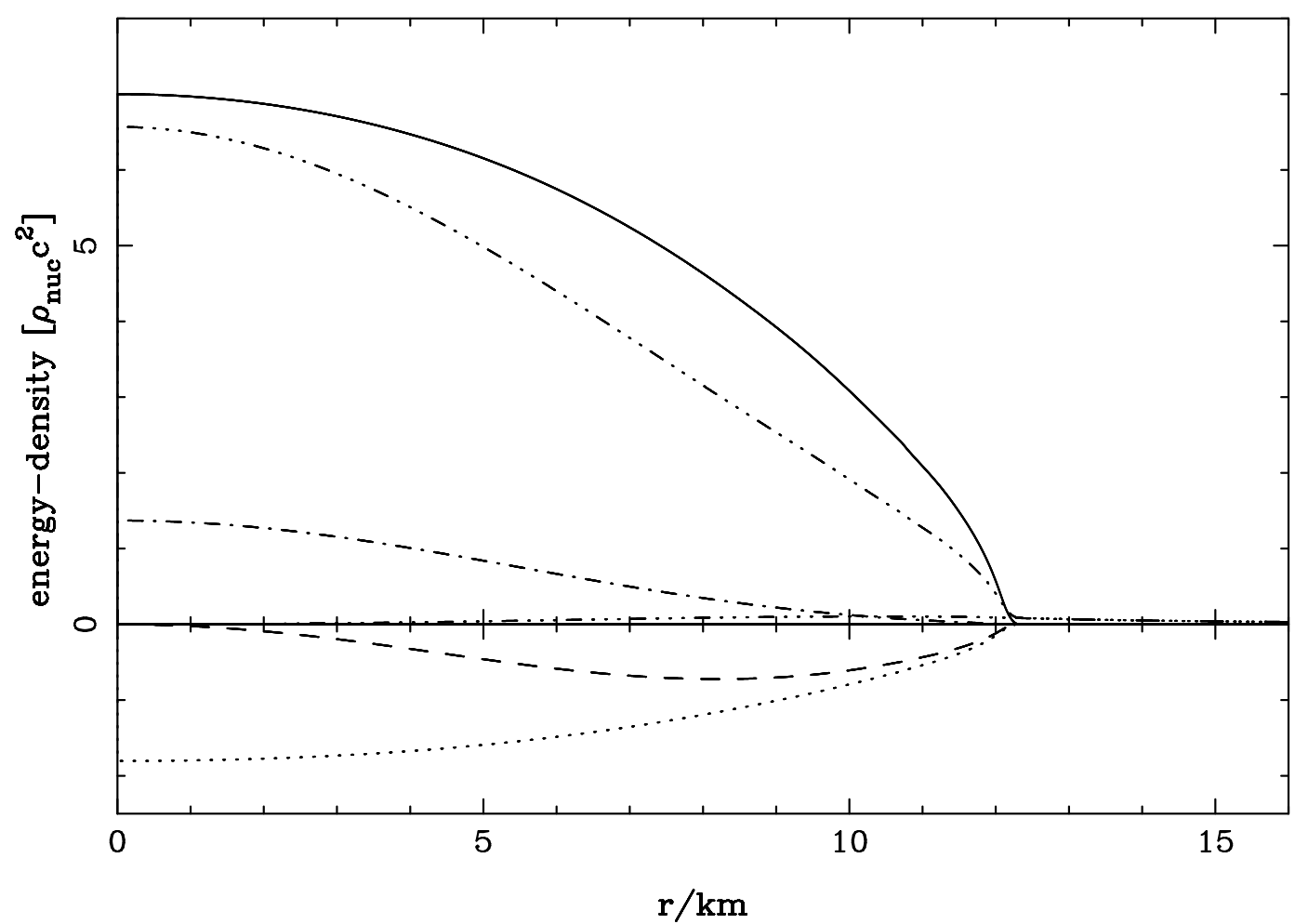

FIG. 5. Energy-densities as a function of $r$ for the same case of Fig. 11 (see the text for description) corresponding to (from the upper to the lower curve respectively) $\rho_{\text {bar }}^{\text {eff }}$ (solid line), $E$ (dash-dotted line; larger values), $\rho_{\text {int }}$ (dash-dotted line; lower values), $E_{\phi}^{\xi}$ (dash-dotted line; lowest positive values), $\rho_{\text {bar }}^{\text {bind }}$ (dashed line), and $E_{\rho}^{\xi}$ (dotted line). In this scale, the energy-density $E_{\phi}$ lies almost on the r-axis. 


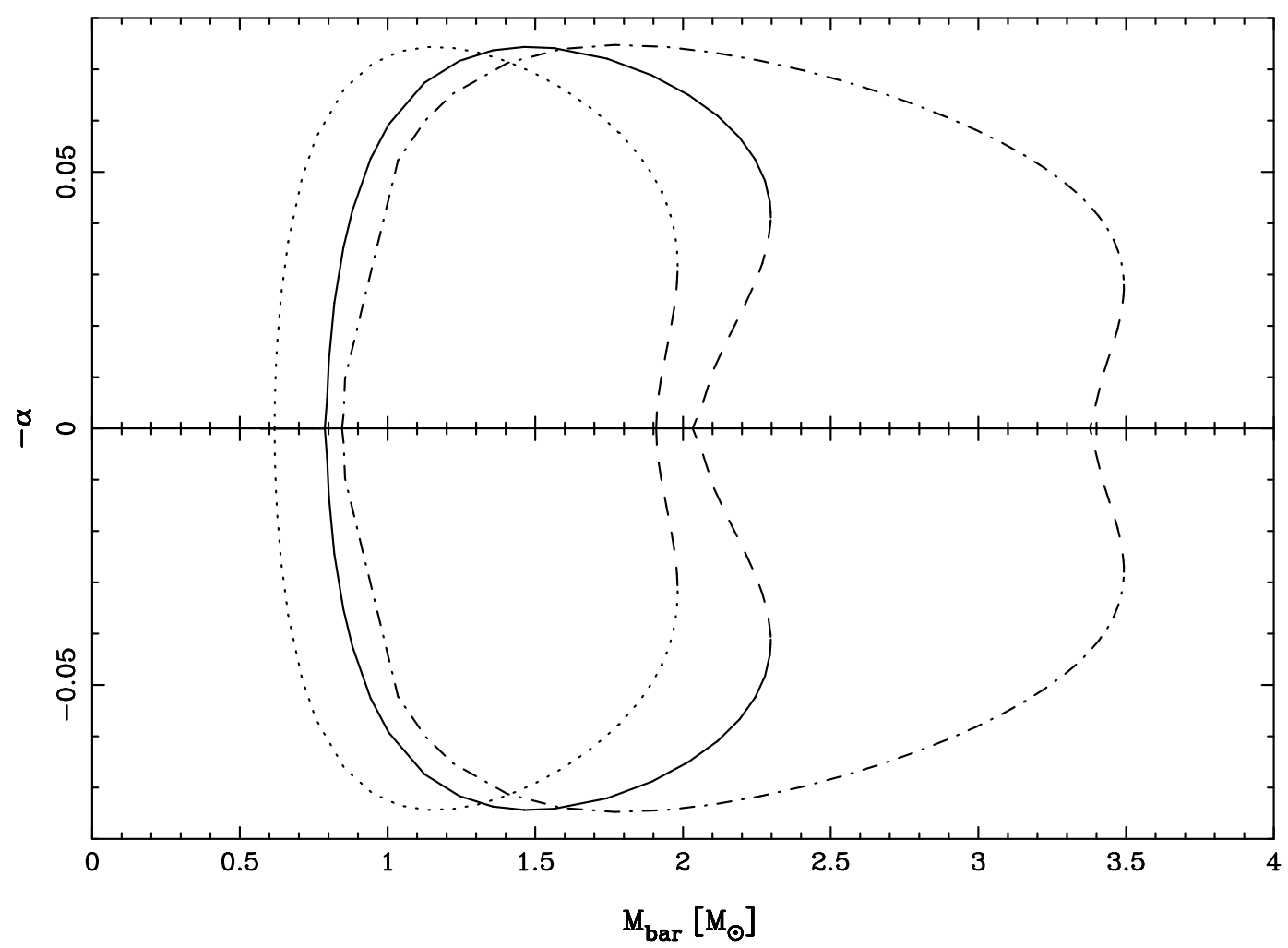

FIG. 6. Coupling strength vs the total baryon mass for the EOS labeled HKP (dash-dotted line), DiazII (solid line), and PandN (dotted line) for $\xi=2$. The dashed segments of the lines refer to the unstable configurations. The positive branches $(-\alpha>0)$ correspond to configurations with $\phi>0$, while the negative ones $(-\alpha<0)$ refer to those with $\phi<0$. The critical baryon masses for spontaneous scalarization are shown in Table 1 . 


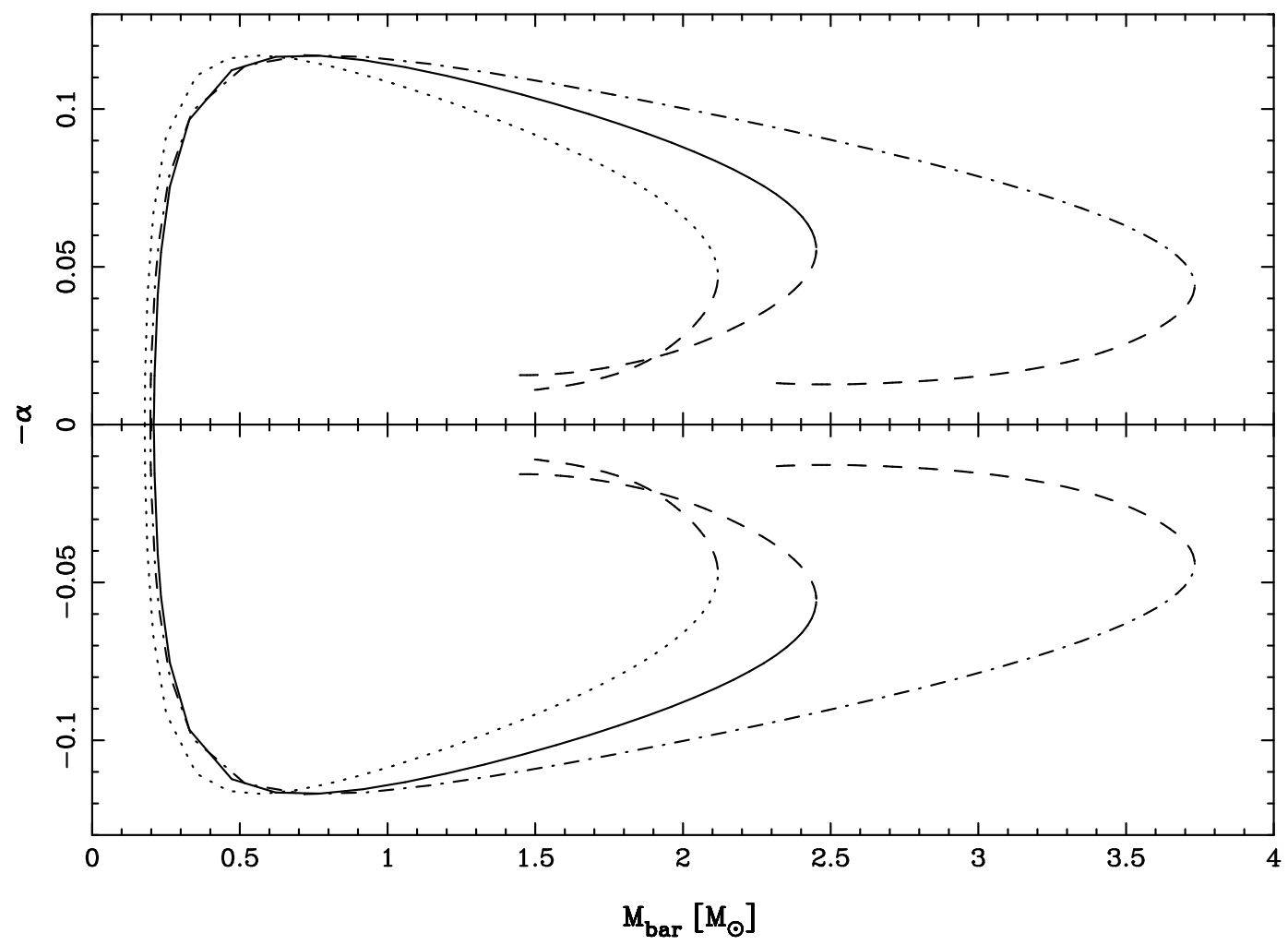

FIG. 7. Similar as Fig. 6 for $\xi=6$. 


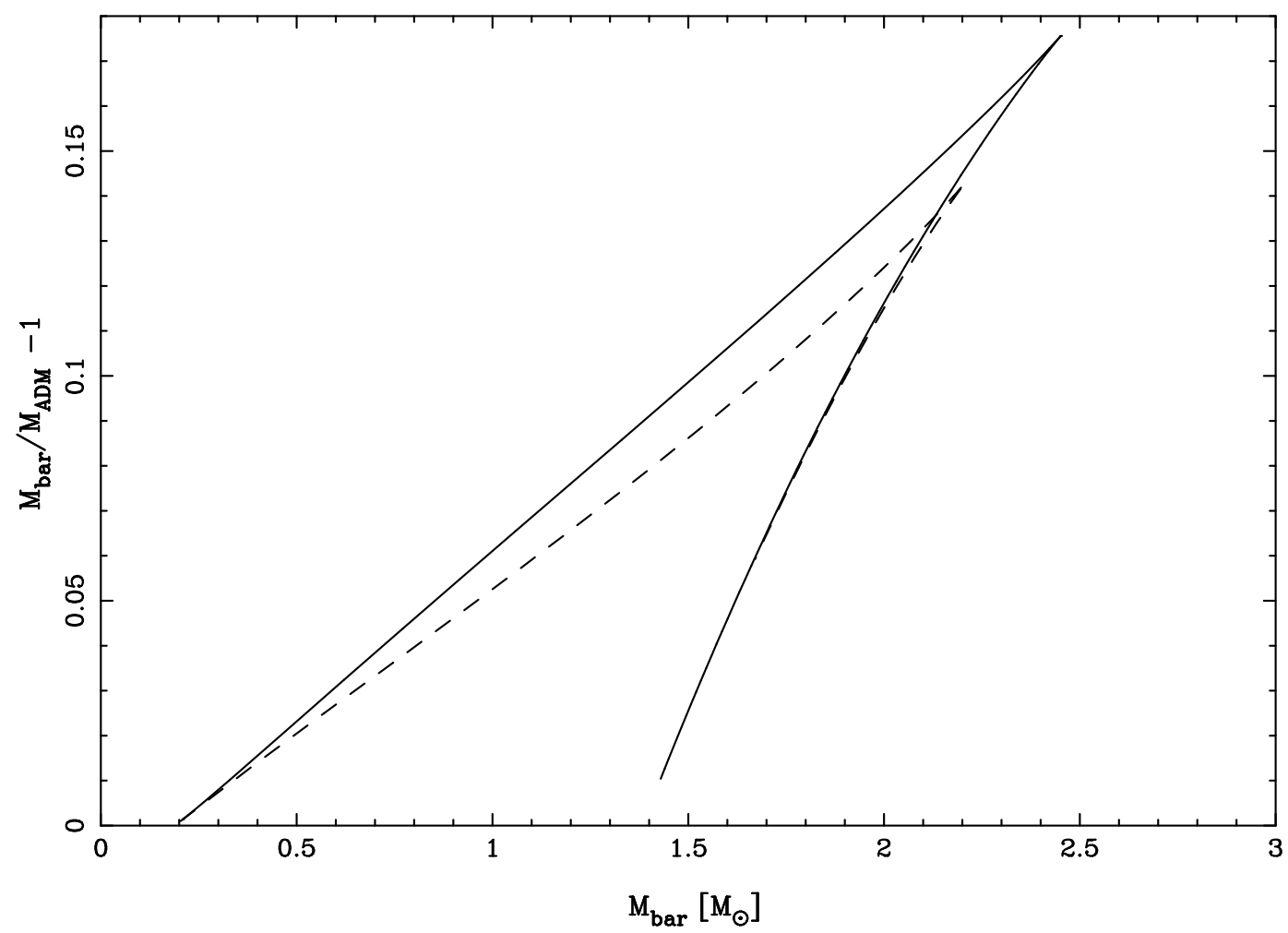

FIG. 8. Total fractional binding-energy as a function of the total baryon mass for the EOS DiazII with $\xi=6$. The solid line refers to configurations computed with $\phi \neq 0$ while the dashed line depicts the configurations with $\phi=0$. Here second branches (unstable configurations) are also shown. Beyond some critical baryon-mass the configurations with $\phi \neq 0$ are energetically more favorable than the corresponding at the same baryon mass with $\phi=0$. Note the increase in the maximum baryon-mass from $2.21 M_{\odot}(\phi=0)$ to $2.45 M_{\odot}(\phi \neq 0)$. 


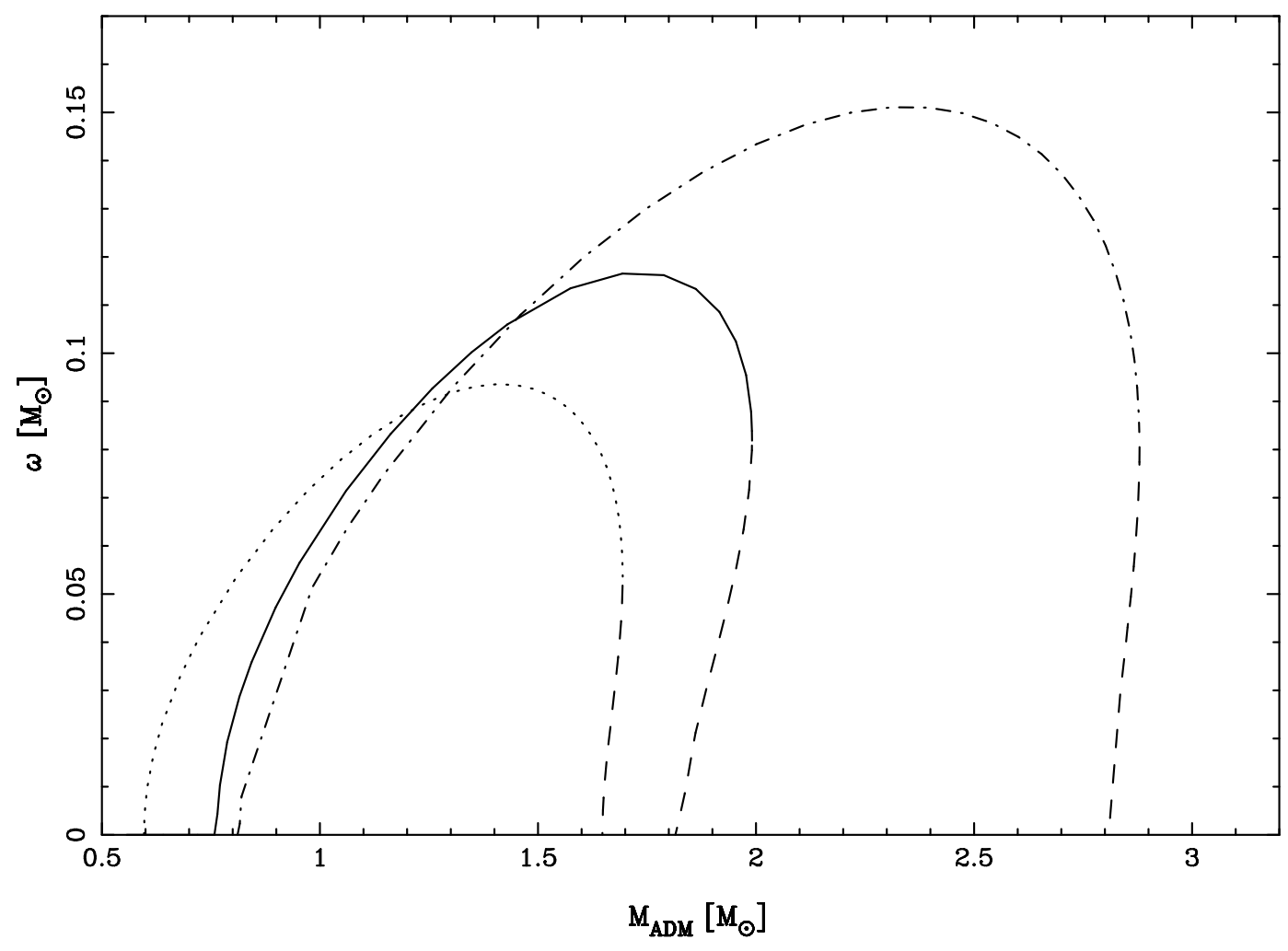

FIG. 9. Scalar charge vs $M_{\mathrm{ADM}}$ for the EOS labeled HKP (dash-dotted line), DiazII (solid line), and PandN (dotted line) with $\xi=2$. The dashed segments of the lines label the unstable configurations. 


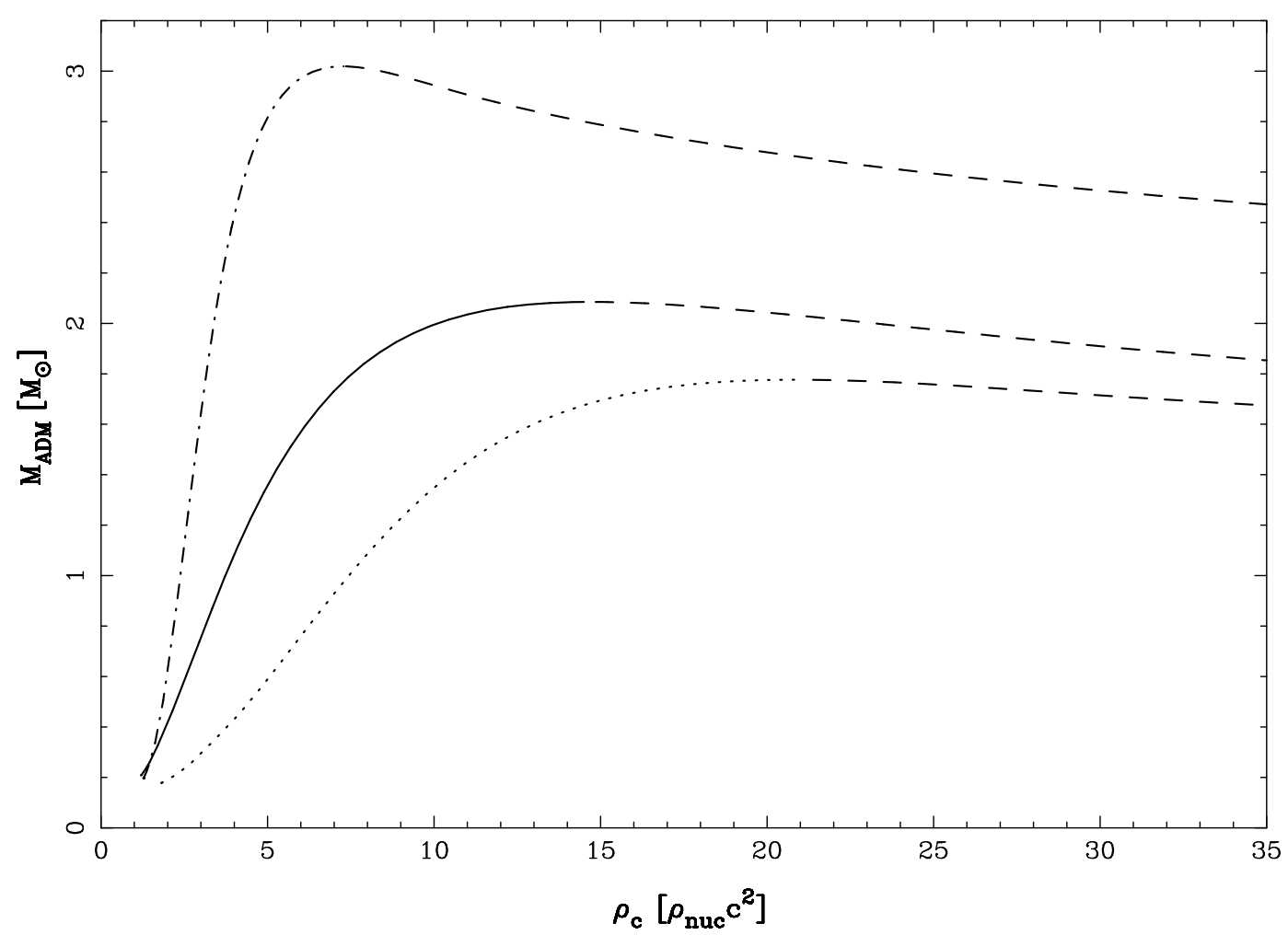

FIG. 10. Total gravitational mass as a function of the central energy-density for the EOS HKP (dash-dotted line), DiazII (solid line) and PandN (dotted line) for $\xi=6$. The dashed segments of the lines correspond to unstable configurations. 


\section{REFERENCES}

[1] T. Damour and G. Esposito-Farèse, Class. Quant. Grav. 9, 2093 (1992).

[2] T. Damour and G. Esposito-Farèse, Phys. Rev. Lett. 70, 2220 (1993).

[3] T. Damour and G. Esposito-Farèse, Phys. Rev. D 54, 1474 (1996).

[4] C.M. Will, Theory and Experiment in Gravitational Physics, (Cambridge Univ. Press, Cambridge 1981).

[5] A. Salmona, Phys. Rev. 54, 1218 (1967)

[6] W. Hilldebrandt and H. Heintzmann, Gen. Relativ. Grav. 6, 663 (1974)

[7] R.A. Saenz, Astrophys. J. 212, 816 (1977)

[8] H.W. Zaglauer, Astrophys. J. 393, 685 (1992)

[9] T. Harada, Progress of Theoretical Physics 98, 359 (1997).

[10] T. Harada, gr-qc/9801049

[11] J. Novak, submitted to Phys. Rev. D, gr-qc/9707041

[12] T. Tsuchida, G. Kawamura and K. Watanabe, gr-qc/9802040

[13] R. Dick, Gen. Rel. Grav. 30, 03 (1998)

[14] D. Sudarsky and R.M. Wald, Phys. Rev. D, 461453 (1992).

[15] B.K. Harrison, K.S. Thorne, M. Wakano and J.A. Wheeler, Gravitation Theory and Gravitational Collapse, University of Chicago Press, 1965

[16] M. Salgado, S. Bonazzola, E. Gourgoulhon and P. Hansel, Astron. Astrophys. 291, 155 (1994).

[17] M. Salgado, PhD Thesis, University of Paris VII, 1994.

[18] J. Díaz-Alonso, Phys. Rev. D 31, 1315 (1985).

[19] V. R. Pandharipande, Nucl. Phys. A 174, 641 (1971)

[20] P. Haensel, M. Kutschera and M. Prózyński, Astron. Astrophys. 102, 299 (1981).

[21] W. Press, B.P. Flannery, S.A. Teukolsky, W.T. Vetterling, Numerical Recipes, The art of scientific computing, Cambridge University Press, 1986, p. 578.

[22] R.M. Wald, General Relativity, University of Chicago Press, 1984.

[23] G. Baym, C. Pethick and P. Sutherland, Astrophys. J. 170299 (1971); G. Baym, H.A. Bethe and C. Pethick, Nucl. Phys. A 175225 (1971) 